\section{Rupture of Jejunal Varices \\ Treated by Percutaneous \\ Transhepatic Obliteration \\ and Endoscopic Injection \\ Sclerotherapy with \\ N-Butyl-2-Cyanoacrylate}

Intestinal variceal bleeding in cirrhotic patients is a life-threatening event. We report here on a case of bleeding intestinal varices which was successfully treated nonsurgically.

A 53-year-old man was admitted on an emergency basis because of massive melena. He had undergone a subsegmentectomy of the liver for hepatocellular carcinoma 8 years previously, and Hassab's operation for esophageal varices at the same time. $\mathrm{He}$ had then had a history of intestinal obstruction due to abdominal adhesion which had been treated surgically. Upper endoscopic examination and colonoscopy did not reveal the bleeding point. Percutaneous transhepatic portography (PTP) revealed intestinal varices which had formed between the jejunal vein and the left renal vein (Figure 1). Percutaneous transhepatic obliteration (PTO) of the varices was performed using coils. After PTO, the flow from the varices diminished and the melena ceased. However, 1 month later, there was a recurrence of melena, and PTP revealed jejunal varices with new inflow routes. A second PTO was performed, but the melena reappeared 1 week later. Computed tomography (CT) revealed portal vein thrombosis; therefore PTO was impossible. The intestinal fiberscope (SIF-10; Olympus, Tokyo, Japan) revealed jejunal varices $10 \mathrm{~cm}$ below the ligament of Treiz, and a fibrin clot which indicated the bleeding point (Figure 2). We decided to inject $1.0 \mathrm{ml}$ of $\mathrm{N}$-butyl-2-cyanoacrylate under endoscopic control. After endoscopic injection sclerotherapy (EIS), the melena ceased and the hemoglobin level was stabilized. No rebleeding episodes have been observed up to 9 months later.

Ectopic varices outside gastroesophageal lesions are less common in patients with portal hypertension [1]. Because of difficulties in diagnosis and treatment, bleeding from jejunal varices is generally massive and life-threatening. Most cases have been treated surgically $[2,3]$. Some reports have suggested that a portosystemic shunt is the definitive therapy for bleeding from intestinal varices [2], but the choice of surgical procedure depends on the severity of the liver disease and extent of intra-abdominal adhesions. In this case, the poor liver function and high degree of intra-abdom-

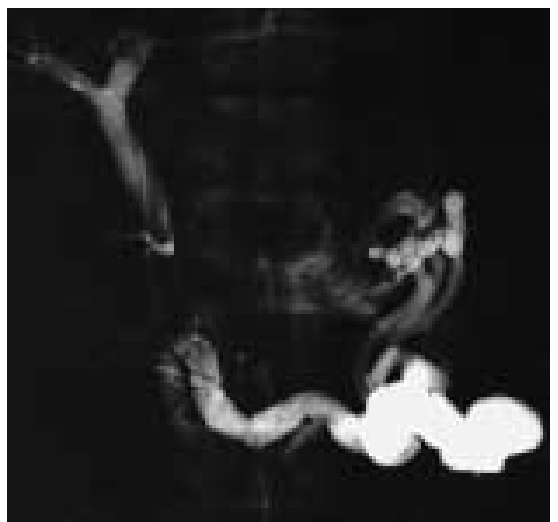

Figure 1 Percutanous transhepatic portography revealed intestinal varices, which were considered to be the source of bleeding

inal adhesion caused us to hesitate before performing surgical treatment. Therefore, we undertook a nonsurgical management of this unusual case.

T. Akahoshi ${ }^{1}$, K. Tanoue ${ }^{1}$, M. Tomikawa ${ }^{1}$, M. Hashizume ${ }^{2}$, K. Sugimachi ${ }^{1}$

${ }^{1}$ Dept. of Surgery II, Faculty of Medicine,

Kyushu University, Fukuoka, Japan

${ }^{2}$ Dept. of Disaster and Emergency

Medicine, Kyushu University,

Fukuoka, Japan

\section{References}

${ }^{1}$ Norton ID, Andrews JC, Kamath PS. Management of ectopic varices. Hepatology 1998; 28: 1154-1158

${ }^{2}$ Yuki N, Kubo M, Noro Y, et al. Jejunal varices as a cause of massive gastrointestinal bleeding. Am J Gastroenterol 1992; 87: $514-517$

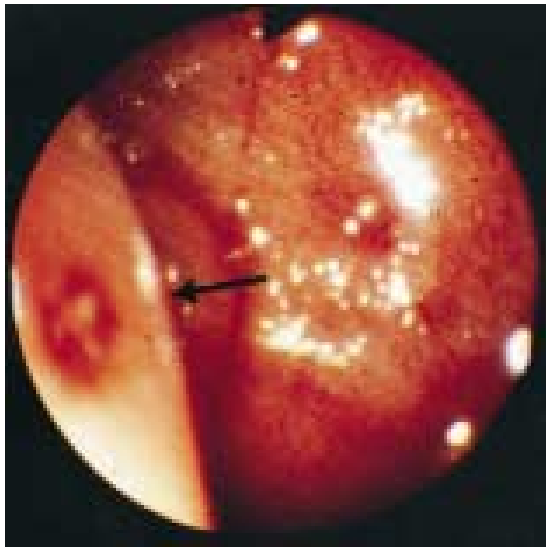

Figure 2 Intestinal endoscopy revealed nodular varices with a fibrin clot (arrow) which was considered to indicate the bleeding point

${ }^{3}$ Migou S, Hashizume M, Kishihara F, et al. Jejunal variceal bleeding after esophageal transection in a patient with idiopathic portal hypertension. Hepatogastroenterology 1998; 45: 503-507

Corresponding Author

T. Akahoshi, M.D.

Dept. of Surgery II

Faculty of Medicine

Kyushu University

3-1-1 Maidashi

Higashi-ku,

Fukuoka 812

Japan

Fax: $\quad$ +81-92-6425482

E-mail: tomohiko@surg2. med.kyushu-u.ac.jp 